\title{
ON A FAMILY OF WIENER TYPE SPACES
}

\section{R. H. FISCHER, A. T. GÜRKANU and T. S. LIU}

University of Massachusetts Department of Mathematics and Statistic Amherst, MA.01003, U.S.A
Ondokuz Mayıs University Faculty of Art and Sciences Department of Mathematics Samsun, Turkey

(Received June 16, 1993 and in revised form June 20, 1994)

ABSTRACT. Research on Wiener type spaces was initiated by N.Wiener in [15]. A number of authors worked on these spaces or some special cases of these spaces. A kind of generalization of the wiener's definition was given by H.Feichtinger in [2] as a Banach spaces of functions (or measures, distributions) on locally compact groups that are defined by means of the global behaviour of certain local properties of their elements. In the present paper we discussed wiener type spaces usinq the spaces $A_{W, \omega}^{p, q}(G)$ and $F_{W, \omega}^{P, q}(G)$ (c.f.[8]) as a local component, and $L_{V}^{r}(G)$ as a global component, where $w$ and $\nu$ are Beurling weights on $G$ and $\omega$ is a Beurling weight on $\hat{G}($ c.f. [13]).

KEY WORDS AND PHRASES. Weighted $\mathrm{L}^{\mathrm{P}}$-spaces, Beurling Algebra, Wiener type space, BF-space.

1991 AMS SUBJECT CLASSIFICATION CODES. 43.

NOTATIONS. Troughout this paper G denotes a locally compact abelian group (non-compact, non-discrete) with the dual group $\hat{G}$. Certain well known terms and their definitions may be found, e.g., in [2], [3], [7], [13], [14], [9], [8]. In the present paper we have used two kinds of Fourier Transforms: The classical Fourier transform(^)(c.f.e.g[13]) and a generalized Fourier transform F (c.f.[4]) discussed below.

Let $Q$ be a fixed open and relatively compact subset of $G$. We define

$$
S_{0}(G)=\left\{f \mid f=\Sigma L_{y_{n}} f_{n}, Y_{n} \in G, f_{n} \varepsilon A_{Q}, n \geq 1 \text { and } \Sigma\left\|f_{n}\right\|_{A}<\infty\right\}
$$

where

$$
A_{Q}=\{h \in A(G) \mid \operatorname{supp}(h) \subset Q\}
$$

and $\mathrm{L}_{\mathrm{y}}$ denotes the translation operator. Any Representation of $f$ in the form (1) is called an admissible representation. Endowed with the norm

$$
\|f\|_{S_{0}}=\inf \left\{\left\|f_{n}\right\|_{A}, f=\Sigma L_{Y_{n}} f_{n} \text { admissible }\right\},
$$


$S_{0}(G)$ is the smallest strong $l_{Y}$ chararter invariant segal algebra on $G$. It is well-known that the Fourier trensforms induce isomorphisms between the spaces $S_{O}(G)$ and $S_{O}(\hat{G})$. The generalized Fourier transform then is defined by

$$
\langle\hat{\sigma}, f\rangle=-\langle\sigma, \hat{f}\rangle \text { for } f \in S_{O}(\hat{G}), \sigma \in S_{O}^{\prime}(G)
$$

It is easy to see that above mentioned qeneralized Fourier transform coincides with the Fourier transform in the sense of tempered distributions for the special case of $G=\mathbb{R}^{\mathrm{m}}$.

Throughout this work, we also will use Beurling weights, i.e.realvalued, measurable and locally bounded functions $w$ on a locally compact abelian group $G$ which satisfy $1 \leq w(x), w(x+y) \leq w(x) w(y)$ for $x, y \in G$. For $1 \leq \mathrm{p}<\infty$, we set

$$
L_{W}^{p}(G)=\left\{f \mid f \cdot W \varepsilon L^{p}(G)\right\} .
$$

Under the norm $\|f\|_{p, w}=\|f \cdot w\|_{p}$, this is a Banach space. When $p=1, L_{w}^{1}(G)$ becomes an algebra under convolution, called Beurling algebra, c.f. [13].

In this paper another two important tools are the spaces $A_{W, \omega}^{p, q}(G)$ and $\quad F_{w, w}^{p, q}(G)$ with the norms $\|\cdot\|_{w, w}^{p, q}$ and $\|\cdot\|_{F}$ respectively [8]. These spaces and the norms are defined as follows:

$$
\begin{gathered}
A_{w, w}^{p, q}(G)=\left\{f \mid f \in L_{w}^{p}(G) \text { and } \hat{f} \varepsilon L_{w}^{q}(\hat{G})\right\}, \\
\|f\|_{w, w}^{p, q}=\|f\|_{p, w}+\|\hat{f}\|_{q, w}
\end{gathered}
$$

and

$$
F_{w, \omega}^{p, q}(\hat{G})=\left(A_{w, w}^{p, q}(G)\right),\|\hat{f}\|_{F}=\|f\|_{w, \omega}^{p, q}
$$

where $F$ is the generalized Fourier transform, $w$ and $\omega$ are Beurling weights on $\mathbf{G}$ and $\hat{G}$ respectively.

The main parts of this work deal with certain Wiener-type spaces in the sense [2]: the definition is the following: Let $B$ be a Banach space. Assume that there exists a homogeneous Banach space $\left(A,\|\cdot\|_{A}\right)$, continuously embedded into $\left(C_{b}(G),\|\cdot\|_{\infty}\right)$, which is a Banach algebra under pointwise multiplication and is stable under complex conjuqation, such that $\left(B,\|\cdot\|_{B}\right)$ is continuously embedded into $A_{C}^{\prime}(G)=\left(A(G) \cap C_{C}(G)\right)^{\prime}$ and also is a Banach module under pointwise multiplication. Here $C_{C}(G)$ is the space of continuous functions with compact support, $A_{C}(G)=A(G)$ $n C_{C}(G)$ is given the (locally convex) inductive limit topology of its subspaces $\left(A_{K}(G),\|\cdot\|_{A}\right), K \subset G$ compact, and $A_{C}^{\prime}(G)$ is the topological dual of $A_{C}(G)$. Let now $B_{10 c}$ be the space of all $f \varepsilon A_{C}^{\prime}(G)$ such that $h f \varepsilon B$ for $h \in A_{C}(G)$; this is a locally convex vector space whose topology is defined by the seminorms $f \rightarrow\|h f\|_{B}, h \in A_{C}(G)$. Fix an open, relatively compact set $Q \subset G$ and define, for $f \in B$ and $x \in G$, the "restriction norm $\|f\|_{B(x+O)}$ of $f$ over $x+Q$ " to be 


$$
\inf \left\{\|y\|_{B} \mid y \in B, h f: \text { hg for } h \in A_{x+Q}(\text { (i) }\}\right. \text {. }
$$

For $f \in B_{10 c^{\prime}}$ set $F_{f}(x)=\|f\|_{B(x+Q)}$. If now $C$ is a solid, translation in variant BFm-space on G, one defines the Wiener-type space W(B.C) by

$$
\left.W(B, C)=\left\{f \varepsilon B_{1 O C} \mid F_{f} \varepsilon C\right\} \text { and }\|f\|_{W(B, C)}-\left\|F_{f}\right\|_{F}\right\} \cdot
$$

Lastly recall that a Banach convolution triple (BCT) is a triple $\left(B^{1}, B^{2}, B^{3}\right)$ of $B F-s p a c e s$ such that convolution, given by

$$
f^{1} \times f^{2}(x)=\int_{G} f^{1}(x-y) f^{2}(y) d y
$$

for $f^{i} \varepsilon B^{i} \cap C_{C}(G)(i=1,2)$, extends to a continuous bilinear nap $B^{1} \times B^{2}+B^{3}$ (of norm 1).

1. THE WIENER-TYPE SPACES $W\left(A_{w, \omega}^{p, q}(G), L^{r}\right)$

The construction of the wiener-type spaces mentioned in the section title requires some preliminary considerations, notably Theorem 1.5 below. First of all we introduce the Banach spaces.

$$
A^{u}(G)=F\left(L_{u}^{1}(\hat{G})\right)
$$

where $u$ is an arbitrary weight function on $\hat{G}$ with the norm $\|\hat{g}\|_{u}\|g\|_{1, u}$ and $F$ is the classical Fourier transform. We $\operatorname{set} A_{C}^{u}(G)=A^{U}(G) \cap C_{C}(G)$ and equip it with the inductive limit topology $\tau_{u}$ of the subspaces $A_{K}(G)=$ $=A(G) \cap C_{K}(G), K \subset G$ compact, equipped with their $\|\cdot\|_{u}$-norms. Since it is obvious that $\tau_{u}$ then is finer than the norm-topology of $A_{C}(G)$, it is Hausdorff and hence the dual space $A_{C}^{u}(G)^{\prime}=\left(A_{C}^{u}(G), \tau_{u}\right)^{\prime}$ separates the points of $A_{C}^{u}(G)$. Note also that the subspaces $A_{K}^{u}(G)$ are closed in $A^{u}(G)$ : Indeed, if $\left(h_{n}\right) \subset A_{K}^{u}(G)$ converges to $h \in A^{u}(G)$ with respect to $\|\cdot\|_{u^{\prime}}$ the

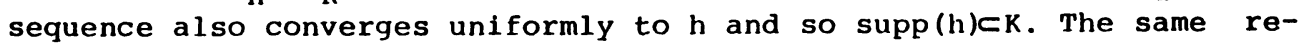
asoning shows that for $K=I, A_{K}^{u}(G)$ is $\|\cdot\|_{u}$-closed in $A_{L}^{u}(G)$. Consequently, if $G$ is o-compact, then $\left(A_{C}^{u}(G), \tau_{u}\right)$ also is complete since then it is a strict inductive limit of Banach spaces.

LEMMA 1.1. If $w_{1}<w_{2}$ and $w_{2}$ satisfies Beurling Domar condition (shortly (BD) i.e $\left.\sum_{n \geq 1} \frac{\operatorname{logw}_{2}\left(t^{n}\right)}{n^{2}}<\infty, t \in \hat{G}\right)$, then $A_{C}{ }^{W_{2}}(G) \subset A_{C}{ }^{1}(G)$ and $A_{C}{ }^{W^{2}}(G)$ is everywhere dense in $A_{C}{ }^{1}(G)$.

PROOF. Since $w_{w_{2}}<w_{2}$ then the inclusion ${\underset{w}{C}}_{C}(G) \subset L_{w_{2}}^{1}(G) \subset L_{w_{1}}^{1}(G)$ is satisfied and $A^{W_{2}}(G)$ is everywhere dense in $A{ }^{1}$ with respect to the norm $\|\cdot\|_{w_{1}}$. Also since $w_{2}$ satisfies (BD) then there is an approximate identity $w_{w_{1}}\left(e_{\alpha}\right)_{\alpha e I} \subset \Lambda_{w_{2}}^{K}(G)$, where $\Lambda_{w_{2}}^{K}=\left\{f \mid f e L_{w_{2}}^{l}(G)\right.$, suppêf compact $\}$. Take any $\hat{g e A}_{c}{ }^{w_{1}}(G)$ and $\varepsilon>0$. There exists a function $\hat{f} \in{ }^{W_{2}}(G)$ such that

$$
\|\hat{f}-\bar{g}\|_{w_{1}}<\frac{\varepsilon}{2}
$$




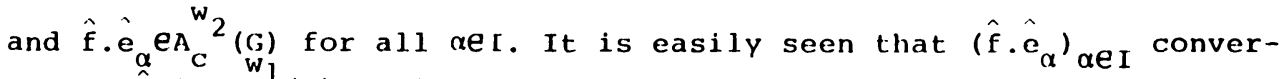
ges to $\stackrel{\alpha}{\hat{g}}$ in $\Lambda_{c}{ }^{w}(G)$. This proves our lemma.

COROI.LARY 1.2 . Let $w_{1}$ and $w_{2}$ be satisfied the hypothesis in lemma 1.1. We endow the space $A_{C}{ }^{W_{1}}(G)$ with the induced inductive limit topology by $A^{W_{1}}(G)$. $A_{C}^{W_{2}}(G)$ is also evervwhere dense in $A_{C}{ }^{W}(G)$ with respect to induced inductive limit topoloqv brcause it is strictlv finer than the oriqinal topology on $A_{C}{ }^{N} l(G)$ which is induced topology by $A^{W}(G)$.

COROLlaRY 1.3. Again let $w_{1}, w_{2}$ be satisfied the hypothesis in lemma 1.1. Using the inclusion $A_{C}{ }^{{ }_{2}}(G) \subset A_{C}{ }^{W}(G)$ and the Corollary 1.2. one obtains that $A_{C}{ }_{C}(G)^{\prime}$ is continuously embedded into $A_{C}{ }^{\prime}(G)^{\prime}$. Specially if ${ }^{w_{1}} \sim w_{2}$ then $\left(A_{C}{ }^{W}(G)\right)^{\prime}=\left(A_{C}^{w_{2}}(G)\right)^{\prime}$.

PROPOSITION 1.4. If $1 \leq p \leq \infty$ and the weight function $w$ on $G$ satis fies $(B D)$ condition then $A_{w, \omega}^{p, q}(G)$ is continuously embedded into $\sigma\left(A_{C}^{W}(G)\right.$, $\left.A_{C}^{(1)}(G)\right)$.

PROOF. It is known that ${ }_{A}^{\mathrm{p}, \mathrm{q}}(\mathrm{G})$ is continuously embedded into $\sigma\left(A_{C}^{\prime}(G), A_{C}(G)\right)[8]$. If one uses the above embedding and the corollary 1.3., easily proves the Proposition.

In order to obtain all the properties of $\lambda^{\mathrm{u}}(\mathrm{G})$, etc., required for the construction of Wiener-type spaces in the sense e.g. of Feichtinger, cf.[2], we assume henceforth that tho weight function $u$ on $\vec{G}$ satisfies (BD) and symmetric and $\omega<u$, where $\omega$ is the second weight function in $A_{w, \omega}^{p, q}(G)$ (For example one can take $u=w(x)+w(-x)$ ).

First of all, $A^{u}(G)$ now satisfies the requirements of [2]: It is clear that $A^{u}(G)$ is continuously embedded into $C_{b}(G)$; in fact, the embedding map has norm $\leq 1$. Moreover, since u satisfies (BD), $A^{U}$ (G) is known to be a wiener space, cf. [13], hence is reqular. It is a Banach algebra under pointwise multiplication and also is translation-invariant: $L_{u}^{1}(\hat{G})$ is character-invariant and that the maps $M_{x}$ are isometries; moreover, for $g \in L^{1}(G), x \rightarrow M_{x} g$ is continuous [8]. By taking Fourier transforms, we now conclude that $A^{u}(G)$ is translation-invariant, translation maps are isometries and that $x \rightarrow L_{x} h$ is continuous from $G$ into $A^{u}(G)$ for each $h \in A^{u}(G)$. In other words: $A^{u}(G)$ is homogeneous Banach space. Lastly, the symmetry of $u$ implies that $A^{u}(G)$ is closed under complex conjuqation.

Secondly $A_{w, w}^{p, q}(G)$ is a pointwise Banach module over $A^{\omega}(G),[8]$. Since $w \leq u$ then $A_{w, w}^{P, q}(G)$ is also a Banach module over $A^{u}(G)$. Consequently one shows

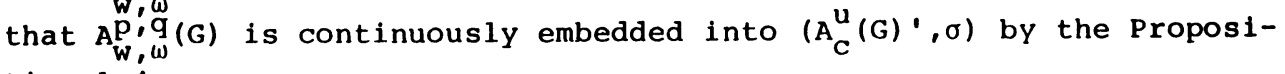
tion 1.4 .

With this, Feichtinqer's general hypotheses are satisfied and the construction of the wiener-type spaces $W\left(A_{W,(u)}^{p, q}(G), C\right), C$ a solid $B F-s p a c e$ proceeds in the standard manner.

Using the arquiments in Theorem 1. in [2] and doing some small modification, the proof of the following theorem is completed. 
THEOREM 1.5. (i) The Wiener type space $W\left(A_{w, w}^{p, q}\left((i), L_{v}^{r}((i))\right.\right.$ is a Banach space under the norm

$$
\|\mathrm{f}\|=\|\mathrm{F}\|_{\mathbf{r}, v^{\prime}}
$$

where $f \in W\left(A_{W, W}^{p, q}(G), L_{V}^{r}(G)\right)$ and

$$
F(z)=F_{f}(z)-\left(\|f\|_{w, w}^{p, q} \mid z+\cap\right), z \in(; \text { and }
$$

QCG is any open subset of $G$ with compact closure. It is also continuously embedded into $\left(A A_{w, w}^{p, q}(G)\right)$ loc

(ii) The set

$$
\Lambda_{0}=\left\{f \in A_{w, \omega}^{p, q}(G) \mid \text { suppf is compact }\right\}
$$

is continuously embedded into $W\left(A_{W, w}^{p, q}(G), L_{v}^{r}(G)\right)$.

$$
\begin{gathered}
\text { (iii) } W\left(A_{W, w}^{p, q}(G), L_{v}^{r}(G)\right) \text { is left(right) invariant, and } \\
\qquad\left\|L _ { x } \left|\left\|\leq\left|\left\|L_{x} \mid\right\|_{W, \omega}^{p, q} \cdot\left\|L_{x}\right\| \|_{r, v^{\prime}}\right.\right.\right.\right.
\end{gathered}
$$

where $\||\cdot|\|,\|\|_{r} \mid \|_{w, w}^{p, q}$ and \|\|$\cdot\|\|_{r, v}$ are operator norms on $W\left(A_{w, w}^{p, q}(G), I_{v}^{r}(G)\right)$, $A_{w, \omega}^{p, q}(G)$ and $L_{v}^{r}(G)$ respectively.

(iv) The translation is strongly continuous in the wiener type spaces $w\left(A_{w, \omega}^{p, q}(G), L_{v}^{r}(G)\right)$.

(v) $W\left(A_{w, w}^{p, q}(G), L_{v}^{r}(G)\right)$ is a Banach module over $: W\left(A(G), L^{\infty}(G)\right)$ with respect to the pointwise multiplication.

PROPOSITION 1.6. Let $p \geq 1$. Then $W\left(A_{w, w}^{p, q}(G), L_{w}^{p}(G)\right)$ is a Banach module over $\left.W\left(A_{W, w}{ }_{w}, G\right), L_{W}^{1}(G)\right)$ with respect to convolution.

PROOF. It is easy to show that every locally compact Abelian group is a IN rgroup (i.e. a group having a compact neighbourhood of identity is invariant under inner automorphisms). It is known that $A \underset{w, \omega)}{p, q}(G)$ is a left(right) convolution module over $A_{w, w}^{l, q}(G)$ [8]. Hence since

$$
\left(A_{w, w}^{1, q}(G), A_{w, w}^{p, q}(G), A_{w, w}^{p, q}(G)\right) \text { and }\left(L_{w}^{1}(G), L_{w}^{p}(G), L_{w}^{p}(G)\right)
$$

are a Banach convolution triples on $G$. Then

$$
\left(W\left(A_{w, w}^{1, q}(G), L_{w}^{l}(G)\right), W\left(A_{w, w}^{p, q}(G), L_{w}^{p}(G)\right), W\left(A_{w, w}^{p, q}(G), L_{w}^{p}(G)\right)\right)
$$

is a Banach convolution triples on $G$ and the inequality

$$
\begin{gathered}
\left\|f \times g \mid W\left(A_{W, w}^{p, q}(G), L_{W}^{p}(G)\right)\right\| \leq \\
\leq\left\|f\left|W\left(A_{W, w}^{l, q}(G), L_{W}^{p}(G)\right)\|\cdot\| q\right| W\left(A_{W, w}^{p, q}(G), L_{W}^{p}(G)\right)\right\|
\end{gathered}
$$

is satisfled by the Theorem 3. in [2] for all $f \in W\left(A_{W, W}^{l, q}\right)(G), L_{W}^{p}(G)$ ) and

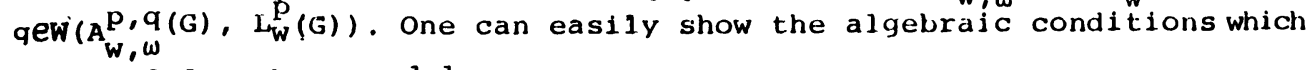
are needed to be a module. 
PROPOSITION 1.7. $W\left(A_{W,(i)}^{p,(G)}, L_{V}^{r}(F)\right)$ is a BF-space on $G$.

PROOF. By the Theorem 1.5. (i), $W\left(A_{W,(1)}^{p, q}(G), L_{V}^{r}(G)\right)$ is continuously embedded into $\left(A_{W, v}^{p, q}(G)\right){ }_{10 c}$. That means given any $h \in A_{c}$ (thus a seminorm

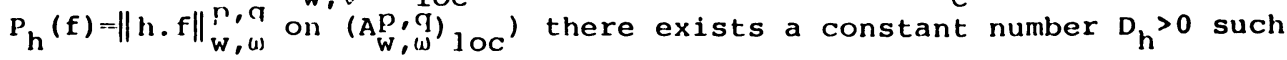
that

$$
\|h f\|_{w, \omega}^{p, q} D_{h}\left\|f \mid W\left(A_{w, w}^{p, q}(G), L_{v}^{r}(G)\right)\right\| .
$$

for all f $W\left(A_{W, w}^{p, q}(G), L_{v}^{p}(G)\right)$. Hence one can write

$$
\|h . f\|_{p} \leq D_{h} \cdot\left\|f \mid W\left(A_{w, w}^{p, q}(G), L_{v}^{r}(G)\right)\right\| .
$$

Also $A^{\omega}(G)$ is a regular Banach algebra with respect to pointwise multiplication, then one may choice a function $\operatorname{heA}_{C}^{\omega}(G)-A^{(\omega)}(G) \cap C_{C}(G)$ satisfying $0 \leq h<1$ and $h(x)=1$ for all $x \in K$. We let supph $K_{0}$. Then $x_{K}(x) \leq h(x)$, hence $x_{K}(x)|f(x)|<h(x)|f(x)|$ for all $x \in G$. Since $L^{p_{\hookrightarrow}} L_{1}^{l}{ }_{1}^{\prime}$, then there exists $D_{K_{0}}>0$ such that

$$
K_{0}^{S}|h(x) f(x)| d x \leq D_{K_{0}} \cdot\|h \cdot f\|_{p} .
$$

Also one has

$$
{ }_{K}^{\int}|f(x)| d x \leq K_{0}^{f}|f(x) h(x)| d x .
$$

The proof is completed combining the formülas (2), (3) and (4).

PROPOSITION 1.8. The wiener type space $W\left(A_{w, \omega}^{p, q}(G), L_{v}^{r}(G)\right)$ is a $B a-$ nach convolution module (left and right because $G$ is an Abelian group) over some Beurling algebra $L_{w_{O}}^{1}(G)$.

PROOF. We proved in Proposition 1.7 that $W\left(A_{W, \omega}^{p, q}(G), L_{v}^{r}(G)\right)$ is a BF-space, thus $W\left(A_{W, \omega}^{P, G}(G), L^{r}(G)\right) \hookrightarrow L_{10 C}^{1}(G)$. BY the Theorem $1.5 \ldots$ this space is left invariant and translation operator in $W\left(A_{W, w}^{p, q}(G), L_{v}^{r}(G)\right.$ is continuous. Now a simple application of vector valued inteqral shows that $W\left(A_{w, w}^{p, q}(G), L_{v}^{r}(G)\right)$ is a Banach module over $L_{w_{0}}^{1}(G)$, where

$$
W_{O}(x)=\max \left(1,\|\| L_{x}\left|W\left(A_{w, w}^{p, q}(G), L_{v}^{r}(G)\right)\right| \|\right) \text {. }
$$

COROLLARY 1.9. $W\left(A_{w, w}^{\mathrm{p}, q}(G), L_{v}^{r}(G)\right)$ is a left(right) Banach module over $L_{v}^{l}(G)$ if $v(x)$ is a weight satisfying $v(x)>w_{O}(x)$ for all $x \in G$, where $w_{0}(x)$ is defined as in Proposition 1.8 .

REMARK. By the Theorem 1.5 (iii), one writes

$$
\left|\left\|L _ { x } \left|\left\|\leq\left|\left\|L _ { x } \left|\left\|_ { w , \omega } ^ { p , q } \cdot \left|\left\|L_{x} \mid\right\|_{r, \nu^{\prime}}\right.\right.\right.\right.\right.\right.\right.\right.\right.
$$

where \|\|$\cdot\|\|,\|\cdot\| \|_{w, w)}^{p, q}$ and \|\|$\cdot \|_{r, v}$ are operator norms on $w\left(A_{w, w}^{p, q}(G), L_{v}^{r}(G)\right)$, $A_{W, \omega}^{p, q}(G)$ and $L_{v}^{r}(G)$ respectively. It is also known that $\left\|L_{x}\right\|_{r, v} \leq \nu(x)$ and $\left|\left\|L_{x} \mid\right\|_{W, \omega}^{p, q} q_{W(x)}[8]\right.$. Then we have 


$$
\left\|\mathrm{L}_{\mathrm{x}} \mid\right\| \leq \mathrm{W}(\mathrm{x}) \cdot v(\mathrm{x}),
$$

for all xeg. Since $w, v$ are weight functions, then the function $w . v$ is locally bounded. Using the inequality(2) it is easily seen that $\mathrm{x} \rightarrow\|\| \mathrm{L}_{\mathrm{x}} \mid \|$ is also looally bounded.

Given a weighted space $L_{w}^{p}(G)$ the associated weighted sequence space is denoted by $\ell^{\mathrm{p}} \mathrm{w}$ and defined

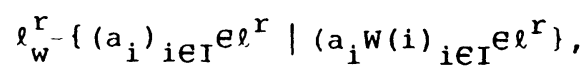

where the discrete weight $W$ given by $W(i)-W\left(x_{i}\right)$ for $i \in I$. It is known that $\ell_{w}^{r}$ is a Banach space with respect to the norm

$$
\|z\|_{r, w}=\left(\sum_{i \in I}\left|a_{i} w(i)\right|^{r}\right)^{\frac{1}{r}},
$$

where $z=\left(a_{i}\right)_{\text {ieI }}$

LEMMA 1.10. For any $z e \ell_{w^{\prime}}^{r} z \neq 0$ the function $z+\left\|L_{p} z\right\|_{r, w}$ is equivalent to the weight function $w$, i.e there is a constant $c>0$ such that one has

$$
c^{-1} W(p) \leq\left\|L_{p} z\right\|_{r, W} \leq c \cdot W(p) .
$$

PROOF. Result can be obtained by a slight modifications of the proof of Lemma 2.2. in [7].

It is also easy to prove the following lemm using the closed graph theorem.

LEMMA 1.11. If $\ell_{w_{1}}^{r_{1}}=\ell_{w_{2}}^{r_{2}}$ then there is some constant c>0 such that

$$
\|z\|_{r_{2}, w_{2}} \leq C \cdot\|z\|_{r_{1}, w_{1}}
$$

for all $z=\left(a_{i}\right)_{i e I} e^{e w_{1}}$

If we use the Lemma 1.10 and Lemma 1.11 easily prove the following lemma:

LEMMA 1.12. $\ell_{w_{1}}^{r} \subset l_{w_{2}}^{r}$ if and only if $w_{2}<w_{1}$.

THEOREM 1.13. Let $\mathrm{U}_{1}$ and $\mathrm{U}_{2}$ be the weight functions in construction of wiener type spaces $W\left(A_{w_{1}, w_{1}}^{p, q}, L_{v_{1}}(G)\right)$ and $W\left(A_{w_{2}, w_{2}}^{p, q}(G), L_{v_{2}}(G)\right)$ respectively. Also assume that $w_{1}, w_{2}, v_{1}, v_{2}$ weights on $G_{;}^{\prime} w_{1}, w_{2}$ weights on $\hat{G}$ and $1 \leq p, q, r_{1}, r_{2}<\infty$. If $u_{1} \sim u_{2} \quad r_{2} \leq r_{1}, u_{1}<u_{2}$ and

$$
A_{w_{2}, w_{2}}^{p, q}(G) \subset A_{w_{1} w_{1}}^{p, q}(G)
$$

then

$$
W\left(A_{w_{2}, w_{2}}^{p, q}(G), L_{v_{2}}^{r_{2}}(G)\right) \hookrightarrow W\left(A_{w_{1}, w_{1}}^{p, q}(G), L_{V_{1}}^{r}(G)\right) .
$$




$$
\begin{gathered}
\text { proof. Since } \lambda_{w_{2},{ }^{\prime},{ }_{2}}^{p, q}(G) \subset A_{w_{1}, w_{1}}^{p, q}(G) \text { then there exists } c>0 \text { such that } \\
\qquad\|\mathrm{f}\|_{w_{1}, w_{1}}^{p, q} \leq C .\|f\|_{w_{2}, w_{2}}^{p, q}
\end{gathered}
$$

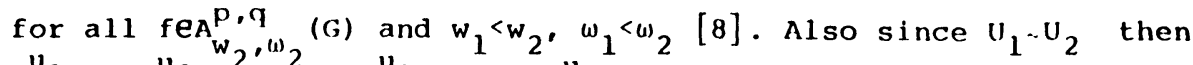
$A_{C}{ }^{u_{1}}(G)=A_{C}{ }^{u_{2}}(G)$ and $\left(A_{C}{ }^{u_{1}}(G)\right)^{\prime}=\left(A_{G}{ }^{\prime(1)}(G)\right)^{\prime}$ by the Lemma 1.1. Hence a simple calculation shows that

$$
\left(\Lambda_{\left.w_{2},{ }^{\prime \prime \prime}\right)}^{\Gamma, q}(G)\right)_{\ell, o c} \leftrightarrow\left(\Lambda_{w_{1},(1)}^{p, q}(G)\right)_{\ell, o c} .
$$

Now using the definition of Wiener-type space, (2) and (3) we have

$$
W\left(A_{w_{2},()_{2}}^{p, q}(G), L_{v_{2}}^{r_{2}}(G)\right) \quad c+W\left(A_{w_{1}, w_{1}}^{p, q}(G), L_{v_{2}}^{r_{2}}(G)\right) .
$$

Also because the Proposition 3.7. In [6] we write

$$
W\left(A_{w_{1}, w_{1}}^{p, q}(G), I_{v_{2}}^{r_{2}}(G)\right) \leftrightarrow W\left(\Lambda_{w_{1}, w_{1}}^{p, q}(G), I_{v_{1}}^{r}(G)\right)
$$

if and only if

$$
\left(L_{v_{2}}^{r_{2}}(G)\right)_{d} \subset\left(L_{v_{1}} r_{1}(G)\right)_{d}
$$

where ${ }_{r_{1}}\left(L_{v_{2}}^{r_{2}}(G)\right) d$ and $\left(L_{v_{1}}^{r}(G)\right)_{d}$ are the discretes of the spaces $L_{v_{2}}^{r_{2}}(G)$ and $L_{v_{1}}{ }_{1}(G)$ respectively. Since $r_{2} \leq r_{1}$ and $v_{1}<v_{2}$ then

$\ell_{u_{2}}^{r_{2}} \subset \ell_{u_{1}}^{r}$. This completes the proof.

It is known that $A_{w_{2},(1)}^{p, q}$ (G) $\subset A_{w_{1},(1)}^{p, q}$ (G) if and only if $w_{1}<w_{2}$ and $\omega_{1}<w_{2}[8]$. If one uses Theorem 1.13 and Lemma 1.12 easily proves the following corollary.

COROLLARY 1.14. Let $u_{1} \sim u_{2}, w_{1}<w_{2}$ and $w_{1}<w_{2}$. Then

$$
W\left(A_{w_{2}, w_{2}}^{p, q}(G), L_{v_{2}}^{r}(G)\right) \leftrightarrow W\left(A_{w_{1}, w_{1}}^{p, q}(G), L_{v_{1}}^{r}(G)\right)
$$

if and only if $v_{1}<v_{2}$.

COROLLARY 1.15. If $w_{1} \sim w_{2}, w_{1} \sim w_{2}, v_{1} \sim v_{2} u_{1} \sim u_{2}$ and $r_{1}-r_{2}$ then

$$
W\left(A_{w_{2}, W_{2}}^{p, q}(G), L_{v_{2}}^{r_{2}}(G)\right)=-W\left(A_{w_{1}, w_{1}}^{p, q}(G), L_{v_{1}}^{r}(G)\right)
$$


2. THE WIENER-TYPE $\operatorname{SPACES~} W\left(\mathrm{~F}_{w, \omega}^{n, q}(G), L_{V}^{r}(\hat{G})\right)$

Let $u$ be a weight function on G. Proceeding as we did in Section 1. we set $A_{C}(G)-A(G)\left\|C_{C}(\hat{G}), A_{C}^{\prime \prime}(\hat{G})-A^{u}(\hat{G})\right\| C_{C}(G)$ equipped with their natural inductive limit topologies, thus the tonology c.q of $A_{C}^{\prime \prime}(\hat{G})$ is the inductive limit $\tau_{\Lambda}$ of subspaces $A_{K}^{u}(G)=A^{u}(G) \cap K_{K}(G), K \subset($; compact, and their $\|\cdot\|_{A}$-topologies. Recall that $\tau_{A}$ is (strictly) finer than the to pology $\tau_{c}$ induced by the usual inductive limit topology of $C_{C}(\hat{G})$.

We assume that the weight function $u$ satisfies(BD) and symmetric and the first weight $w$ in $A_{w, \omega}^{p, q}(G)$ satisfies the condition $w<u$.

Since $W$ is symmetric then $\quad F_{w, w}^{p, q}(\hat{G})=A_{(1), W}^{(T, P)}(\hat{G})$, (see [8]).

Now all conditions are satisfied required for the construction of wiener-type space $W\left(F \underset{w, G)}{p, q}(\hat{G}), L_{v}^{r}(\hat{G})=W\left(A, q, p(\hat{G}), L_{v}^{r}(\hat{G})\right)\right.$. If one uses the properties of the space $A A_{(, I, W}(\hat{G})$ obtains all properties of the wienertype space $W\left(F_{W, \omega}^{P, q}(\hat{G}), L_{V}^{r}(\hat{G})\right)$ like to that of $W\left(A_{w,(i)}^{p, q}(G), L_{V}^{r}(G)\right)$ in Section 1 .

ACKNOWLEDGEMENT. The authors want to thank H.G. Feichtinger for his significant sugqestions regarding this paper. The first named author wants to thank R.H.Fischer and T.S. Liu and University of Massachusetts for their hospitality.

\section{REFERENCES}

1. DOMAR, Y. Harmonic analysis based on Certain commutative Banach algebras, Acta Mat 95, (1956) (1-56).

2. FEICHTINGER, H.G. Banach convolution algebras of Wiener-type:Colloqua Mathematica Societatis Janos Balyai, 35. Functions,series, operators, Budapest (Hungary), 1980 .

3. FEICHTINGER, H.G. On a new Segal algebra, Mh. Math. 92.(1981), (269-289).

4. FEICHTINGER, H.G. Un espace de Banach distributions temperees sur les grupes localement compacts abelians, C.R. Acad. Sci.Paris, 290. (1980) (791-794).

5. FEICHTINGER, H.G. On a class of convolution algebras of functions Ann. Inst.Fourier, Grenoble 27, 3(1977) 135-162.

6. FEICHTINGER, H.G. and GROCHENIC, K.H. Banach spaces related to integrable group representations and their atomic decompositions 1., Journal of functional analysis Vol. 86, No.2 (1989).

7. FEICHTINGER, H.G., and GURKANLI, A.T. On a family of weighted convolution algebras, Internat. J.Math. and Math.Sci. Vol.13, No. $3,(1990)$.

8. FISCHER, R.H., GURKANLI, A.T., and LIU, T.S. On a family of weigh ted spaces, (submitted to the slovaca Math. for publication).

9. FOURNTER, J.J.F., and STEWART, J. Bulletin of the A.M.S., Vol. 13, Number $1,1985(1-21)$.

10. GULICK, S.L., LIU, T.S., and VAN ROOtJ, A.C.M. Group algebra modules 2, The Canadian Journal of Mathematics, Vol.19 (1967) $(151-173)$. 
11. GURKANLI, A.T. Some results in the weighted $A_{p}\left(\mathbb{R}^{n}\right)$ spaces, Demonsratio Mahtematica, Vol. 19/4 (1986), (825-830).

12. LIU, T.S., VAN ROOIJ, A., and WANG, J.K. On some group algebra, modules related to Wiener's algebra M, Pacific J.Math. (55) (1974), (507-520).

13. REITER, H. Classical Harmonic Analysis and locally compact groups., Oxford University Press, Oxford, (1968).

14. WANG, II.G. Homogeneous Banach algebras, Marcel Dekker Inc.(1977).

15. WIENER, N. Tauberian theorems, Ann. of Math. 33(1932), (1-100). 


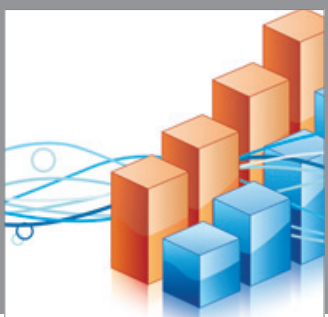

Advances in

Operations Research

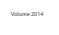

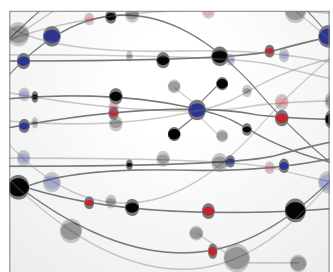

\section{The Scientific} World Journal
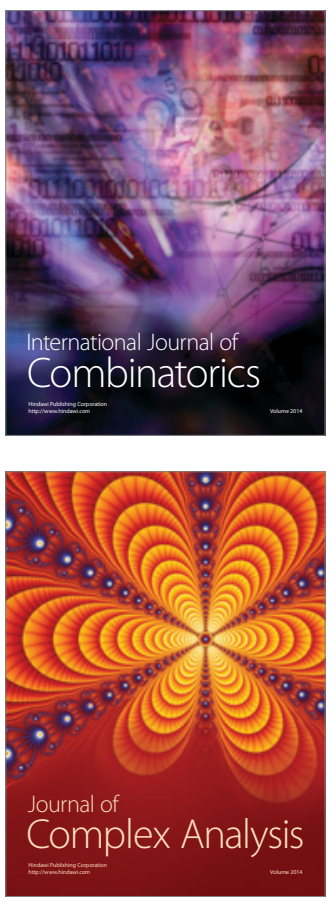

International Journal of

Mathematics and

Mathematical

Sciences
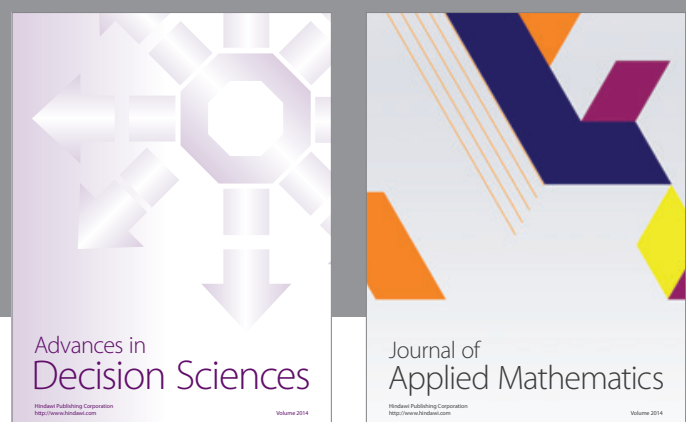

Journal of

Applied Mathematics
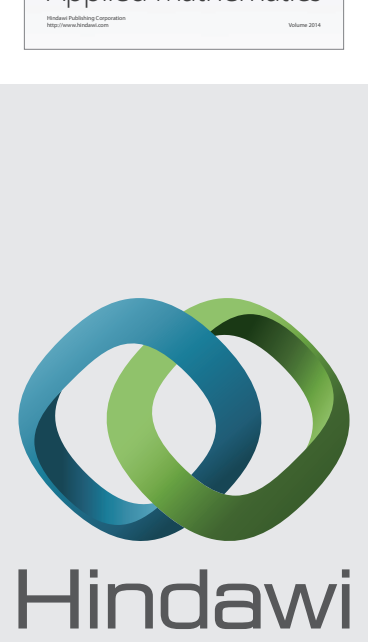

Submit your manuscripts at http://www.hindawi.com
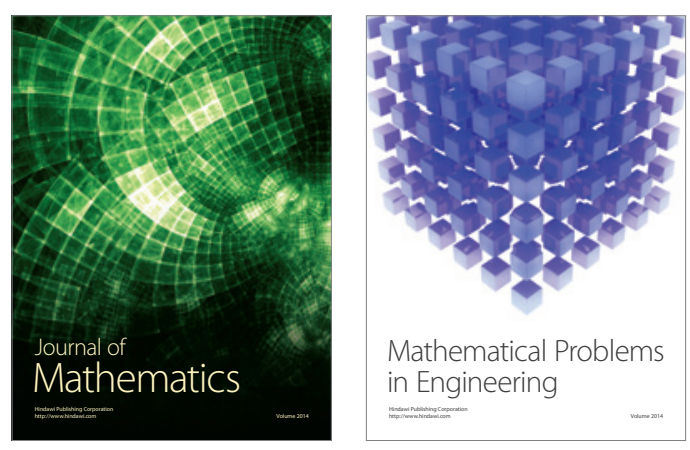

Mathematical Problems in Engineering
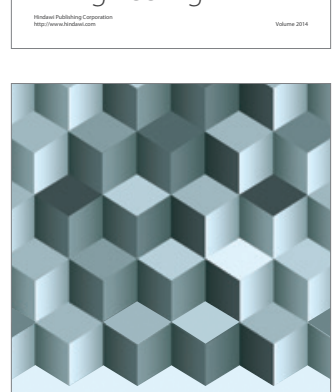

Journal of

Function Spaces
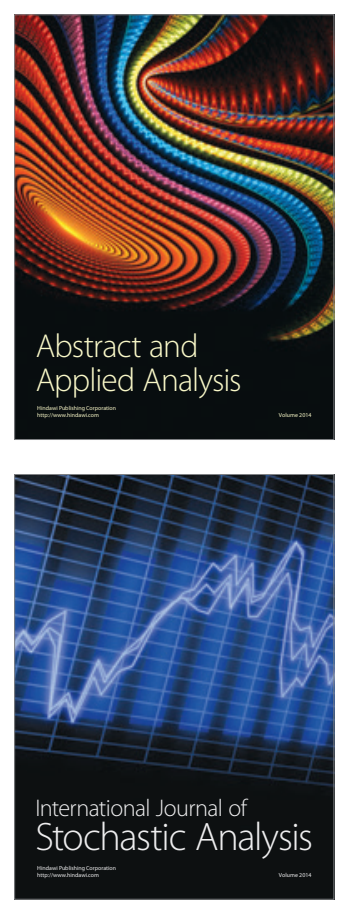

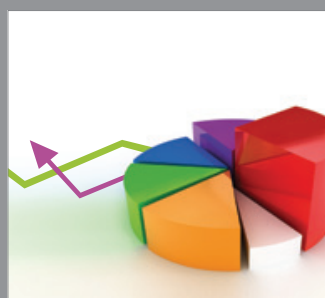

ournal of

Probability and Statistics

Promensencen
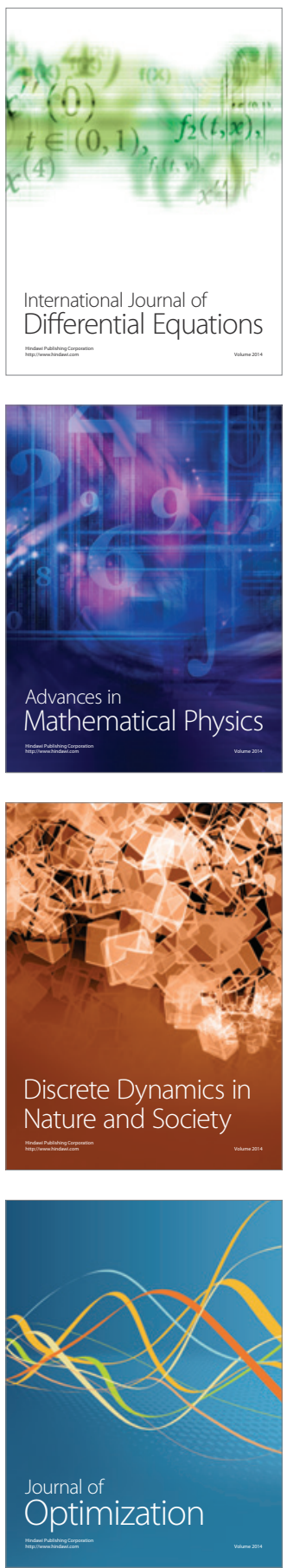